\title{
Outcomes of induction in trial of labor after cesarean delivery
}

\author{
Anubha Varshney ${ }^{1}$, Zehra Mohsin ${ }^{2 *}$ \\ ${ }^{1}$ Department of Obstetrics and Gynecology, GGSH Hospital, New Delhi, India \\ ${ }^{2}$ Department of Obstetrics and Gynaecology, JNMCH AMU, Uttar Pradesh, India
}

Received: 27 December 2018

Accepted: 30 January 2019

\section{*Correspondence:}

Dr. Zehra Mohsin,

E-mail: drzehram@hotmail.com

Copyright: $\odot$ the author(s), publisher and licensee Medip Academy. This is an open-access article distributed under the terms of the Creative Commons Attribution Non-Commercial License, which permits unrestricted non-commercial use, distribution, and reproduction in any medium, provided the original work is properly cited.

\section{ABSTRACT}

Background: The objective of this study is to evaluate the outcomes of induction of labor in women attempting trial of labor after cesarean delivery and to compare maternal and neonatal morbidity and mortality in women who were induced to those delivering spontaneously.

Methods: The prospective study was carried out in the Department of Obstetrics and Gynecology in collaboration with the Neonatal Section, Department of Pediatrics at J.N. Medical College and Hospital, AMU Aligarh. The sample included 280 women with one previous cesarean section, of whom 130 women underwent induction of labor (study group) and 150 were admitted with spontaneous onset of labor. Prostaglandin gel and intracervical Foley's were used for cervical ripening in the study group. Indication of cesarean section, mode of delivery, maternal and neonatal outcome were studied in between groups.

Results: Overall rate of vaginal delivery after cesarean section was $45.3 \%$ and $56 \%$ in both study and control group respectively. The rate of cesarean section were higher in women who were induced and having unfavorable cervix. Maternal and neonatal morbidity were not significantly higher as compared in both groups, however one case of scar rupture was found in study group.

Conclusions: Induction of labor in women with previous cesarean section had higher rates of cesarean section however it does not adversely affect neonatal and maternal morbidity. Overall vaginal birth is safe and effective in women with previous cesarean section by prostaglandin gel or intracervical Foley's. Authors cautiously suggest, induction of labor should be considered in preselected patient with strict monitoring.

Keywords: Cephalopelvic disproportion, Induction of labor, Lower segment cesarean section, Trial of labor after cesarean, Vaginal birth after cesarean

\section{INTRODUCTION}

Vaginal birth after cesarean section (VBAC) has always been a domain of controversies. The dictum "once a caesarean, always a caesarean" has largely permeated the obstetric practice for most of the twentieth century and today. ${ }^{1}$ However, over the past three decades a dramatic rise in cesarean deliveries has been occurring. In 2010, reports from the National Centre for Health Statistics (NCHS) states that the cesarean delivery accounts for
$32 \%$ of all births in the United States. ${ }^{2}$ India is also not excluded from this trend. Though estimated cesarean rates in India in the year 1998 was $7.1 \%$ and the increase in rates annually in India is $16.7 \%$, which is among the highest. ${ }^{3}$ The rise in cesarean rates over the period is still unexplained because of lack of proper data. In India the safe deliveries are $47.6 \%$ and the emergency obstetric care which is provided to the patient is not uniform over the geographical spread. According to the recent NHFS4 (2015-16), the average rate of cesarean section is $17.2 \%$ 
in India which is ranging from $5.8 \%$ in Nagaland to $58 \%$ in Telangana. The trend of cesarean deliveries which was analyzed from 1992-93 to 2015-16 shows that there has been upward trend in cesarean section rates in India. ${ }^{4}$ Some reasons accounting for the increase in cesarean rates are repeat cesarean delivery, delay in childbirth and reduced parity, forceps deliveries and advanced fetal monitoring technique.

Therefore, in order to reduce the overall cesarean delivery rate, certain strategies are required that may prevent the primary cesarean delivery, improve the uptake of VBAC and improve planned VBAC success. It has been estimated that increased uptake of planned VBAC could decrease the overall cesarean section rate by around $5 \% .^{5}$ In a 2010 consensus conference, the National institutes of Health (NIH) examined the safety and outcome of trial of labor after cesarean (TOLAC) and VBAC and factors associated with decreasing rates. The NIH panel had recognized that TOLAC was a reasonable option for many women who had prior cesarean delivery and called all organizations to facilitate access to TOLAC. ${ }^{6}$

Multiple cesarean section has been shown to be associated with higher rates of maternal morbidity and mortality. With each successive caesarean section, the overall morbidity rises continually specifically for major morbidity from triad of placenta previa, placenta accreta and hysterectomy. It is important to decrease the primary cesarean section rate as well as to increase the uptake of VBAC. The problem arises when the pregnant women with previous cesarean section do not go into spontaneous labor and then the need for induction arises or it may be done for other indications like hypertensive disorders, intrauterine growth restriction, and cholestasis of pregnancy.

Induction of labor is a method to help initiate the increase in the rate of contractions in women who are near the end of their pregnancy. Labor induction is generally performed when potential benefits outweigh the risks of continuing the pregnancy. ${ }^{7}$ The purpose of this study is to know the outcome of induced labor in previous cesarean section and to improve our understanding that if there is repeat cesarean section in induced patients, what are causes and what factors determine if a cesarean section or vaginal delivery is more likely.

\section{METHODS}

Authors conducted a prospective cohort study of all singleton pregnancies with history of previous cesarean section who attempted vaginal delivery in department of obstetrics and gynecology in our college JNMCH from September 2015 to October 2017.

A total of 280 women with previous cesarean section were recruited for the study. After counselling 130 patients with previous LSCS were induced for different indications (study group) and 150 women (control group) were left for spontaneous delivery. The following demographic and obstetric variables were recorded maternal age, gravida, parity, gestational age, indication of prior cesarean section, duration of previous cesarean section, indication for induction of labor. Intrapartum characteristics indication of cesarean section, interval between induction to onset of labor, induction to delivery interval, mode of delivery, maternal and neonatal outcome were studied.

The patients were selected from patient attending antenatal clinic, outpatient department (OPD), and patients admitted in hospital. All patients with CPD/ Classical or $\mathrm{T}$ incision/ nonvertex presentation/ IUGR/ severe preeclampsia/ diabetes in pregnancy/ increased neonatal birth weight were excluded from study. On admission pelvic adequacy and bishop score was detected, non-stress test was done. Patients with bishop score $<4$ were induced.

\section{Protocol}

Prior to induction of labor, all patients in study group received a detailed explanation of procedure and written informed consent was provided. According to our local protocol, vaginal prostaglandin gel E2 (cerviprime gel) were considered as first line of agent for cervical ripening followed by intracervical Foley's catheterization, or with both. At the end of study, data was compiled, and results critically analyzed. Stastical analysis was performed by the X2 (Chi Squire) test and ANOVA TEST. A p value of $<0.05$ was considered to be statistically significant.

\section{RESULTS}

Total 130 pregnant women with previous one lower segment cesarean section were underwent induction. The cases in present study ranged from 19- 35 years in age. Mean age of women undergoing induction in our group was $26.14+3.56$ years.

The period of gestation ranged from 37- 42 weeks in present study. Majority of cases presented in gestational age of $39-42(59.2 \%)$ week in present study. Mean gestational age for induction in present study was $39.2+1.2$ week. Maximum induction was executed between 39-42 weeks of gestation. Maximum failed VBAC $(63.6 \%)$ occurred in gestational age of $39-42$ weeks. These patients (study group) were matched with a group in which women with previous section were not induced (control group). There was no statistical difference between the study and control group when comparing maternal age, gravidity, gestational age and birth weight.

The most common indication for induction was for postdated pregnancy $46 \%$. The second most common indication for induction was intrahepatic cholestasis of pregnancy $35 \%$, which was followed by gestational hypertension $8 \%$, IUD in previous pregnancy $7 \%, \mathrm{Rh}$ 
negative pregnancy 3\%. Emergency repeat cesarean sections $38(63.3 \%)$ were higher in post-dated group with vaginal deliveries in 22 cases $(36.7 \%)$. Women which were induced for IHCP, $24(52.1 \%)$ cases had repeat cesarean section and $22(66.7 \%)$ cases had successful TOL (Table 1).

Table 1: Relationship between indication for induction and outcome of labor.

\begin{tabular}{|l|l|l|l|l|}
\hline \multirow{2}{*}{ Indication of induction } & \multicolumn{3}{l}{ Outcome of labor } \\
\cline { 2 - 5 } & LSCS & \multicolumn{2}{l|}{ VBAC } \\
\hline & N & \% & N & \% \\
\hline Post dated & 38 & 63.3 & 22 & 36.7 \\
\hline IHCP & 24 & 52.1 & 22 & 47.9 \\
\hline Gest HTN & 4 & 36.4 & 7 & 63.6 \\
\hline Prev IUD & 3 & 33.3 & 6 & 66.7 \\
\hline Rh negative pregnancy & 2 & 50 & 2 & 50 \\
\hline
\end{tabular}

However, the association was found to be stastically not significant $(\mathrm{p}>0.05)$. Table 2 signify that maximum induction $121(93.1 \%)$ was required in women with unfavourable cervix i.e. bishop score is between 0-4. Women with bishop score between $0-4$ had higher rates of LSCS $66(54.5 \%)$.

Table 2: Relationship between Bishop score and outcome of labor in study group.

\begin{tabular}{|l|l|l|l|l|}
\hline \multirow{2}{*}{ Bishop score } & LSCS & VBAC & \\
\hline $0-4$ & $\mathbf{N}$ & $\mathbf{\%}$ & $\mathbf{N}$ & $\%$ \\
\hline$>4$ & 66 & 54.5 & 55 & 45.5 \\
\hline
\end{tabular}

The rate of successful TOL was $45.5 \%$. While women with bishop score $>4$ had repeat cesarean sections in $55.6 \%$ cases and vaginal deliveries were in $44.4 \%$ cases. The result was found to be non-significant. The mean bishop score was $2.88+1.18(24.6 \%)$. The mean interval from induction to delivery interval was $24.8+11.5$ hours. $24.6 \%$ women had delivery within 12 hours. $30 \%$ of maximum deliveries occurred within 12.1 to 24 hours. $26.9 \%$ delivered within 24.1 to 36 hours and $18.5 \%$ in more than 36 hours.

Table 3: Distribution of cases according to the indication in second cesarean section in study group, $(\mathbf{n}=130)$.

\begin{tabular}{|l|l|l|l|l|}
\hline \multirow{2}{*}{ Bishop score } & \multicolumn{2}{|c|}{ LSCS } & \multicolumn{2}{l|}{ VBAC } \\
\hline $0-4$ & n & \% & n & \% \\
\hline$>4$ & 66 & 54.5 & 55 & 45.5 \\
\hline
\end{tabular}

The results were found to be non-significant $(p>0.05)$. The most common indication of repeat cesarean section was fetal distress $39 \%$. The second most common indication $28 \%$ was non-progress of labor (NPOL). In $23 \%$ cases cesarean was done for scar tenderness, $10 \%$ underwent cesarean section for non-reassuring CTG (Table 3).

Table 4: Outcome of trial of labor in women with previously scarred uterus in both study group and control group.

\begin{tabular}{|l|l|l|l|l|}
\hline Outcome of labor & \multicolumn{2}{|c|}{ Study group } & \multicolumn{3}{|c|}{ Control group } \\
\hline & $\mathbf{N}$ & $\mathbf{\%}$ & $\mathbf{N}$ & $\mathbf{\%}$ \\
\hline VBAC & 59 & 45.4 & 84 & 56 \\
\hline LSCS & 71 & 54.6 & 66 & 44 \\
\hline Total & 130 & 100 & 150 & 100 \\
\hline $\mathrm{X}^{2}$ & 1.108 & & 2.161 & \\
\hline P & $>0.05$ & & $<0.05$ \\
\hline
\end{tabular}

In the study group $45.4 \%$ cases had successful trial of labor while in control group vaginal deliveries were higher i.e. in $56 \%$ cases. Cesarean section rates were higher in the women requiring induction, $54.6 \%$ while it was $44 \%$ in women who underwent spontaneous labor. (Table 4) In present study the comparison was done between study and control group regarding maternal morbidity. The rate of maternal complications was $9.2 \%$ (12 out Of 130) in study group and $3.9 \%$ (6 out of 150) in control group. No cases of maternal mortality were found in both groups. $5.4 \%$ cases in study group had previous scar dehiscence as compared to $2.6 \%$ in control group. Only one case of scar rupture was found in study group in the woman who had successful VBAC after induction with prostaglandin followed by augmentation with oxytocin (Table 5).

Table 5: Comparison between maternal complications in study group and control group.

\begin{tabular}{|l|l|l|l|l|}
\hline \multirow{2}{*}{ Maternal complication } & \multicolumn{2}{c}{ Study group } & \multicolumn{2}{c|}{ Control group } \\
\cline { 2 - 5 } & $\mathbf{N}$ & $\mathbf{\%}$ & $\mathbf{N}$ & $\mathbf{\%}$ \\
\hline Scar dehiscence & 07 & 5.4 & 4 & 2.6 \\
\hline Scar rupture & 01 & 0.7 & Nil & 0.0 \\
\hline PPH & 03 & 2.3 & 2 & 1.3 \\
\hline Endometriitis & 01 & 0.8 & Nil & 0.0 \\
\hline Mortality & Nil & 0.0 & Nil & 0.0 \\
\hline Total cases & 12 & 9.2 & 06 & 3.9 \\
\hline \begin{tabular}{l}
$($ p $>0.05)$ \\
\hline
\end{tabular} & & & & \\
\hline
\end{tabular}

Table 6: Comparison of fetal complication between study group and control group.

\begin{tabular}{|l|l|l|l|l|}
\hline & \multicolumn{3}{c}{$\begin{array}{c}\text { Study } \\
\text { Neonatal complication }\end{array}$} & \multicolumn{2}{c|}{$\begin{array}{c}\text { Control } \\
\text { group }\end{array}$} & group \\
\hline Apgar score less than 7 in 5 min & 06 & 4.6 & 06 & 4.0 \\
\hline Birth asphyxia & 04 & 3.1 & 03 & 2.0 \\
\hline Nicu admission & 10 & 7.7 & 07 & 4.7 \\
\hline Neonatal resuscitation & 00 & 0.0 & 00 & 0.0 \\
\hline Total cases & 20 & 15.4 & 16 & 10.7 \\
\hline
\end{tabular}

The difference between maternal complication was found to be statistically not significant in both groups. The rate 
of neonatal complications was almost similar in study (15.4\% - 20 babies out of 130) and control group (10.7\% 20 babies out of 150 group).7.7\% babies in study group and $4.7 \%$ babies in control group had NICU admission. $3.1 \%$ babies in study and $2 \%$ babies in control group had birth asphyxia. However, the difference between neonatal morbidity was found to be non-significant $(p>0.05)$ in both group (Table 6).

\section{DISCUSSION}

The world is currently noticing a higher rate of an increase cesarean section in both developed and developing countries. Over the past three decades the overall cesarean rates have been steadily rising. A substantial proportion of this increase in cesarean was due to unnecessary operations attributable to nonevidence-based indications, maternal request, professional convenience and over mediatisation of childbirth. Another reason is the reluctance in giving trial of labor to the women with previous cesarean section as there is a risk of uterine rupture which can pose a threat to mother and fetus and due to possibility of subsequent litigations. ${ }^{4}$ The policy of VBAC is a contribution towards bringing down the increasing rate of cesarean section and can help in reducing maternal morbidity and mortality. The trial of labor is a relatively safe procedure, but it should be attempted with caution as it is not risk free. The induction of labor is the common obstetric practice which is done in interest for mother and fetus. One fourth of women undergoing a TOL after cesarean section require an induction of labor. The current success rate of such process is $67 \%$, yet it is considered to be as one of the tool $\mathrm{s}$ for reducing increasing rate of recurrent cesarean section. $^{8}$ Approximately $15 \%$ labors are induced. Labor induction in an unfavorable cervix is different and lengthy procedure and is also tiring for both the mother as well as for obstetrician. The different methods are used for cervical ripening are pharmacological methods like PGE1, PGE2, oxytocin and non-pharmacological methods like Foley catheter, laminaria, amniotomy etc. ${ }^{8}$ In present study 121 cases had an unfavorable Modified Bishop's score (0-4) in study group. Out of these $66(54.5 \%)$ had repeat cesarean section and $55(45.5 \%)$ had a successful TOL. The results were found to be statistically not significant. Kashif S. in their study demonstrated that the women who are presenting with established labor had a greater chance of successful VBAC. ${ }^{9}$ In present study the comparison was done between study and control group regarding maternal morbidity. In present study the rate of maternal complications was $9.2 \%$ (12 out of 130) in study group and $3.9 \%$ (6 out of 150) in control group. No cases of maternal mortality were found in both groups. Only one case of scar rupture was found in study group in the woman who had successful VBAC after induction with prostaglandin followed by augmentation with oxytocin. Eran Ashwal et al have found no significance difference between maternal complication in study and control group. ${ }^{10}$ The neonatal morbidity was examined by noting the incidence of birth asphyxia, NICU stay in neonate born after TOL. The rate of neonatal complication was almost similar in both groups. Eran Ashwal et al have found no significance difference between neonatal complication in study and control group, thus stating that inaction of labor did not adversely affect neonatal and maternal outcome. ${ }^{10}$ In the current study authors aimed to assess pregnancy outcome and patient safety after labor induction in women with previous single low transverse incision. Present study had several key findings:

- Women whose labor was induced were at increased risk for cesarean delivery mainly due to fetal distress.

- Induction of labor after cesarean section was not associated with an increased rate of maternal complications.

- Induction of labor in women with previous cesarean section was not associated with an increased rate of short-term neonatal complications.

In present study about half of the patient with previous cesarean section who were induced underwent cesarean section. The reason for a low rate of success of TOL in present study may be a small sample size, presence of considerable number of unbooked cases in present study who had no and lack of proper fetal monitoring. As postdates was the commonest indication for induction in our series, Authors would recommend that all women with a previous cesarean section should have a routine dating scan to avoid induction for erroneous date.

\section{CONCLUSION}

A successful TOL in the following pregnancy after a primary cesarean section is important in determining a woman's obstetric future. VBAC offers advantages in terms of lowering cesarean section rates, it enhances patient satisfaction and facilitates faster recovery after giving birth. Our experience in this study proves that in women with previous cesarean section, induction of labor is associated with increased risk of failed TOLAC. Induction of labor is not associated with an increased risk of severe maternal or neonatal morbidity. Labor induction in women with previous section is successful in about half of the cases. Induction is a safe and useful tool that can be used as an alternative in women who undergo elective repeat cesarean section especially if it is done in under close maternal and fetal monitoring and in properly selected patients.

\section{Funding: No funding sources Conflict of interest: None declared \\ Ethical approval: The study was approved by the Institutional Ethics Committee}

\section{REFERENCES}

1. Bangal VB, Giri PA, Shinde KK, Gavhane SP. Vaginal birth after cesarean section. North American Journ of medical sciences. 2013;5(2):140. 
2. Menacker F, Hamilton BE. Recent trends in cesarean delivery in the United States. US Department of Health and Human Services, Centers for Disease Control and Prevention, National Center for Health Statistics; 2010.

3. Singh G, Gupta ED. Rising incidence of caesarean section in rural area in Haryana, India: a retrospective analysis. Inter $\mathbf{J}$ Gynecol Obstet. 2013;17(2):1-5

4. Radhakrishnan T, Vasanthakumari KP, Babu PK. Increasing Trend of Caesarean Rates in India: Evidence from NFHS-4. JMSCR. 2017;5(8):2616776.

5. Al Qahtani N, Al Borshaid S, Al Enezi H. Induction of Labor with PGE2 after One Previous Cesarean Section: 18 Years' Experience in a University Hospital. International Journal of Clinical Medicine. 2011;2(1):35.

6. Cunningham FG, Bangdiwala SI, Brown SS, Dean TM, Frederiksen M, Hogue CR, King T, Lukacz ES, McCullough LB, Petit NF, Probstfield JL. National Institutes of Health Consensus Development Conference Statement: Vaginal Birth After Cesarean Section: New Insights March 8-10, 2010. Obstetric Anesthesia Digest. 2011;31(3):140-2.
7. Nguyen DT. Retrospective Study on Factors that may Contribute to Cesarean Delivery in Induced Women.

8. Ziyauddin F, Hakim S, Beriwal S. The transcervical foley catheter versus the vaginal prostaglandin e2 gel in the induction of labour in a previous one caesarean section-a clinical study. Journal of clinical and diagnostic research: JCDR. 2013;7(1):140.

9. Kashif S, Mansoor M, Tariq R, Tahira T. Vaginal birth after caesarean section; to evaluate factors for successful outcome. Professional Medical J. 2010;17(4).

10. Ashwal E, Hiersch L, Melamed N, Ben-Zion M, Brezovsky A, Wiznitzer A, et al. Pregnancy outcome after induction of labor in women with previous cesarean section. The J Maternal-Fetal Neonat Med. 2015;28(4):386-91.

Cite this article as: Varshney A, Mohsin Z. Outcomes of induction in trial of labor after cesarean delivery. Int J Reprod Contracept Obstet Gynecol 2019;8:1076-80. 